\title{
Outcome of Nonadherence to Antimicrobial Treatment Guidelines in Ambulatory Patients with Acute Cystitis: A Nationwide Population-based Study
}

\author{
Hsiu-Chen Lin ${ }^{1-3}$, Hsiu-Li Lin ${ }^{4,5}$, Li-Hsuan Wang ${ }^{6,7}$, Chien-Yeh Hsu ${ }^{4}$ and Yu-Mei Hsueh ${ }^{1,8}$
}

\begin{abstract}
Objective The recurrence rate after acute cystitis treatment failure with inappropriate antimicrobials remains unclear. The goal of this study was to explore the relationship between cystitis recurrence and nonadherence of antimicrobial prescriptions to national guidelines using a nationwide population-based data set.

Methods This was a retrospective longitudinal observational cohort study that was conducted using the clinical records of the National Health Insurance Research Database in Taiwan from 2006-2007. After excluding patients younger than 18 years of age, with concurrent infections, urinary tract anomaly, and no antimicrobial treatment, the remaining study population included 36,395 patients with acute cystitis. We evaluated the hazard ratio (HR) of cystitis recurrence within 28 days between the adherence and nonadherence groups using Cox proportional hazard regression analysis.

Results Adherence to antimicrobial prescription guidelines was the factor that most strongly influenced acute cystitis recurrence, with a HR of 0.91 (95\% confidence interval 0.87-0.95) after adjusting for all variables. The incidence rate of acute cystitis in patients receiving antimicrobials adherent to national guidelines was 59.78 per 10,000 person-days. The results of the likelihood ratio test indicated that age, sex, and guideline nonadherence were significant risk factors for recurrent cystitis. The percentage of first-generation cephalosporin prescription was $31.61 \%$, making it the most frequently prescribed guideline-recommended drug. Conclusion Acute cystitis patients with or without multiple chronic comorbidities should be treated with antimicrobials that adhere to recommended guidelines to attain a better therapeutic outcome.
\end{abstract}

Key words: cystitis, guideline, adherence, antimicrobial agents, recurrence, outcome

(Intern Med 53: 1933-1939, 2014)

(DOI: 10.2169/internalmedicine.53.1308)

\section{Introduction}

Urinary tract infections (UTIs) are a common medical condition, accounting for more than 7 million ambulatory visits in the United States annually (1). Cystitis is the most common bacterial UTI that requires antimicrobial therapy in ambulatory patients. Although young, healthy women gener- ally have anatomically and physiologically normal urinary tracts, recurrent cystitis is common in this population (2). A study estimated that the incidence of cystitis among sexually active young women is 0.5 per person per year (3). Approximately $20 \%$ of young women with an initial episode of cystitis have subsequent infections and require empirical antimicrobial therapy (1).

Several studies have demonstrated that recurrence, treat-

\footnotetext{
${ }^{1}$ School of Public Health, College of Public Health and Nutrition, Taipei Medical University, Taiwan, ${ }^{2}$ Department of Pediatrics, School of Medicine, College of Medicine, Taipei Medical University, Taiwan, ${ }^{3}$ Department of Laboratory Medicine, Taipei Medical University Hospital, Taiwan, ${ }^{4}$ Graduate Institute of Biomedical Informatics, College of Medical Science and Technology, Taipei Medical University, Taiwan, ${ }^{5}$ Department of Neurology, General Cathay Hospital, Sijhih Branch, Taiwan, ${ }^{6}$ Department of Pharmacy, Taipei Medical University Hospital, Taiwan, ${ }^{7}$ School of Pharmacy, College of Pharmacy, Taipei Medical University, Taiwan and ${ }^{8}$ Department of Public Health, School of Medicine, College of Medicine, Taipei Medical University, Taiwan

Received for publication July 6, 2013; Accepted for publication March 9, 2014
}

Correspondence to Prof. Yu-Mei Hsueh, ymhsueh@tmu.edu.tw 
ment failure, and increased pathogen resistance may occur from the inappropriate antimicrobial treatment of many infectious diseases. However, very few studies have discussed the recurrence rate of acute cystitis due to nonadherence of antimicrobial treatment to national guidelines, although it has been shown that recurrent cystitis can travel to other sites and lead to other complications, such as pyelonephritis, urosepsis, and even more severe diseases such as renal scarring (4). Therefore, acute cystitis should be appropriately treated during the first visit.

Inappropriate antimicrobial prescriptions or overuse can result in antimicrobial resistance, a phenomenon that has become an increasingly well-known public health problem worldwide $(5,6)$. In particular, countries where antibiotics are commonly available without guidelines are more likely to encounter uropathogen resistance patterns $(7,8)$. The prevalence rates of resistance among Escherichia coli and other uropathogens that cause cystitis have significantly increased $(9,10)$. The cure rate for cystitis varies from 61$82 \%$ in women (3). Because the antimicrobial susceptibility profiles of cystitis-causing uropathogens are highly variable, antimicrobial treatment guidelines for lower UTIs have been established in many countries. These guidelines are based on evidence- and consensus-based recommendations, including common etiologic pathogens, knowledge of the antimicrobial susceptibility profile of uropathogens in the community, and agents that have already been marketed in local regions. Therefore, antimicrobial prescriptions that are adhere to national guidelines can be defined as appropriate prescriptions.

Several studies have evaluated factors associated with local physicians' adherence to UTI treatment guidelines in many health care settings. However, to the best of our knowledge, no study has explored the relationship between cystitis recurrence and adherence of antimicrobials to regional treatment guidelines. Inappropriate antimicrobial prescriptions may intensify microorganism resistance and result in poor outcomes in patients with acute cystitis. Therefore, considering the varied uropathogen resistance patterns, selecting antibiotics that adhere to guidelines will reduce the burden of cystitis, thereby lowering its consequences and costs. The goal of this study was to explore the relationship between cystitis recurrence and nonadherence of antimicrobial prescriptions to national guidelines using a nationwide population-based dataset.

\section{Materials and Methods}

\section{Database}

This was a retrospective cohort study that used the clinical records of the National Health Insurance Research Database (NHIRD) in Taiwan from 2006 to 2007. The database was obtained from the Longitudinal Health Insurance Database 2005 (LHID 2005) of the National Health Insurance (NHI) Research Database. The study population comprised $1,073,891$ patients $(4.7 \%)$ randomly sampled from the
$22,717,053$ people enrolled in the NHI program. There were $22,770,383$ residents in Taiwan in 2005 , and the NHI program covered the medical care of $99.8 \%$ of these citizens. The claims dataset is one of the largest and most comprehensive nationwide population-based datasets available worldwide and includes diagnostic codes of individual diseases, pharmaceutical prescriptions, and medical expenditures on outpatient and inpatient care since 1995. The database was created by the Taiwan National Health Research Institute (NHRI) using a systematic sampling method to randomly select a representative sample of all enrollees. There were no statistically significant differences in demographic characteristics, including age, gender, and distribution between the sample and original populations (11). Because the LHID 2005 dataset consisted of de-identified and secondary data released to the public for research, the study was exempted from full review by the Institutional Review Board of Taipei Medical University.

\section{Study sample/case identification}

Our study sample included all ambulatory patients who obtained treatment for cystitis in this dataset, with the principal diagnosis of these patients being International Classification of Diseases, Ninth Revision (ICD-9-CM) codes 595.0 or 595.9. The study period was from January 1, 2006 to December 31, $2007(n=82,940)$. We restricted the study population to adult patients over 18 years of age. To exclude patients with recurrent cystitis, we excluded patients who were diagnosed with the same ICD-9-CM codes within 3 months prior to the date of their ambulatory care visit. Patients with urinary tract anomalies (ICD-9-CM codes 591-594), such as cystocele, were excluded because they were at risk for recurrent cystitis. We only included the first visit for cystitis treatment if a patient had more than one visit during the study period. Patients with acute cystitis and no antimicrobial treatment were also excluded because they did not receive the studied treatment. Additionally, ambulatory patients who visited for concurrent infectious diseases were excluded from our study due to the possibility of concurrent antimicrobial prescriptions (12). In total, our study population included 36,395 patients. We defined cystitis recurrence as ICD-9-CM code 595.0 or 595.9; other sites of UTI as ICD-9-CM codes 590.3, 590.9, 597.8, or 599.0; pyelonephritis as ICD-9-CM codes 590.0, 590.1, 590.2, or 590.8; and urosepsis as ICD-9-CM code 038.9. Poor outcome was defined as patients who experienced illness recurrence within 28 days of their first visit with acute cystitis (inpatient care and outpatient claims data) (13). Antimicrobial prescriptions adherent to guidelines were defined according to the antimicrobial treatment guidelines of acute cystitis established by the Infectious Diseases Sociality of Taiwan (IDST) (14). This guideline was announced and bulletined on the Department of Health (DOH) website (15). If patients received more than two antimicrobials for acute cystitis or disobeyed the guidelines, we defined it as "nonadherence to guidelines of antimicrobial treatment." 


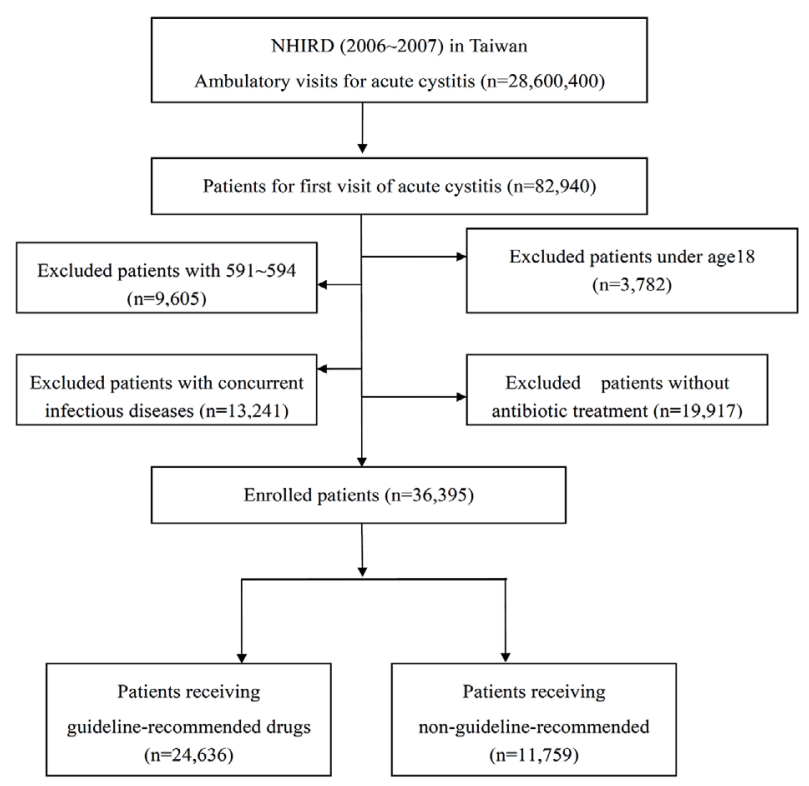

Figure 1. Flowchart of the study subjects recruited from the National Health Insurance Research Database.

\section{Statistical analysis}

The SAS 9.1 statistical package (SAS Institute Inc., Cary, USA) was used for all analyses in this study. Student $t$-tests and $\chi^{2}$ tests were conducted to compare continuous variables and the association of variables, including age, sex, and potential confounders. These included diabetes, chronic obstructive pulmonary disease (COPD), renal disease, other cardiovascular diseases (e.g., hypertension, coronary heart disease, congestive heart disease), diagnosis of the first UTI at less than 15 years of age, urinary incontinence, and contraceptive usage between guideline-recommended drugs and non-guideline-recommended drugs during the follow-up period. Cox proportional hazard regressions were performed to estimate the hazard ratio (HR) of acute cystitis recurrence after adjusting for co-morbidities. Due to the possible colinearity of those comorbidities, we inspected these comorbidities by the multicollinearity method using variance inflation factors. We used the likelihood ratio test to determine the significant risk of a particular variable in the presence of recurrent cystitis and to determine the interaction between variables. In addition, we calculated the 28-day recurrence rate and evaluated the differences in recurrence risk between the two groups by the log-rank test. $\mathrm{p}$ values less than 0.05 were considered statistically significant.

\section{Results}

The LHID2005 database had complete information about cystitis and thus offered a unique opportunity to evaluate antimicrobial treatment and the cystitis recurrence rate. A total of 28,600,400 ambulatory visits for acute cystitis were found in 2006-2007 in the NHI database, and 36,395 pa-
Table 1. Demographic Characteristics of Patients with or without Antimicrobial Adherence to Guidelines in 2006-2007 $(n=36,395)$

\begin{tabular}{|c|c|c|c|c|c|}
\hline \multirow[t]{2}{*}{ Variables } & \multicolumn{2}{|c|}{$\begin{array}{l}\text { Adherence to Guidelines } \\
\qquad(\mathrm{n}=24,636)\end{array}$} & \multicolumn{2}{|c|}{$\begin{array}{c}\text { Nonadherence to } \\
\text { guidelines }(\mathrm{n}=11,759)\end{array}$} & \multirow[t]{2}{*}{$\mathrm{p}$ value } \\
\hline & $\mathrm{n}$ & $\%$ & $\mathrm{n}$ & $\%$ & \\
\hline Mean age (SD) (year) & \multicolumn{2}{|c|}{$47.03(18.18)$} & \multicolumn{2}{|c|}{$47.62(18.05)$} & 0.01 \\
\hline Gender (male) & 3,826 & 15.53 & 2,302 & 19.58 & $<0.001$ \\
\hline Diabetes & 2,034 & 8.26 & 1,013 & 8.61 & 0.25 \\
\hline $\mathrm{COPD}^{\mathrm{a}}$ & 25 & 0.10 & 10 & 0.09 & 0.63 \\
\hline Renal disease & 347 & 1.41 & 155 & 1.32 & 0.49 \\
\hline Urinary incontinence & 1,180 & 4.79 & 504 & 4.29 & 0.03 \\
\hline $\begin{array}{l}\text { Other cardiovascular } \\
\text { diseases }\end{array}$ & 4,660 & 18.92 & 2,327 & 19.79 & 0.04 \\
\hline $\begin{array}{l}\text { Having a first UTI } \\
\leq 15 \text { years-old }\end{array}$ & 48 & 0.19 & 24 & 0.20 & 0.85 \\
\hline Contraceptive usage & 420 & 1.70 & 217 & 1.85 & 0.34 \\
\hline Recurrent cystitis & 3743 & 15.19 & 1988 & 16.91 & $<0.001$ \\
\hline
\end{tabular}

tients remained after excluding patients younger than 18 years age, with concurrent infections, with urinary tract anomalies, and those not receiving antimicrobial treatment (Fig. 1). Table 1 shows the significant differences found in the recurrence rate between patients with antimicrobials adherent to guidelines compared to those with nonadherent antimicrobials. In addition, gender and rates of concurrent comorbidities, such as cardiovascular diseases and urinary incontinence, were significantly different between these two groups. However, there were no significant differences with regard to the other comorbidities (Table 1).

The incidence rate per 10,000 person-days and multivariate adjusted HRs during the 28-day follow-up for acute cystitis recurrence are shown in Table 2. The incidence rate of acute cystitis in patients receiving antimicrobials adherent to national guidelines was 59.78 per 10,000 person-days. However, its adjusted HR was 0.89 [95\% confidence interval (CI) 0.85-0.95] compared to the nonadherent group. The adjusted HR showed that women and elderly patients had a higher incidence of recurrence than male patients and younger patients $(\mathrm{p}<0.01)$. All of the comorbidities had noncollinearity and variance inflation factors. The results of the likelihood ratio test indicated significant associations of recurrent cystitis with the following variables: age, sex, and guideline nonadherence. Antimicrobial prescriptions that adhered to the guidelines resulted in significantly lower recurrence rates than nonadherent prescriptions, even after adjusting for age, gender, other comorbidities, and contraceptive usage. In addition to age and sex, adherence of antimicrobial treatment to the guidelines was also one of the important influencing factors in acute cystitis recurrence after adjusting for all variables.

The log-rank test showed that patients with cystitis who were prescribed guideline-adherent antibiotics had significantly lower recurrence rates during the 28-day follow-up period than those who received nonadherent prescriptions ( $p$ $<0.001$ ) (Fig. 2). Among the total number of antibiotic pre- 
Table 2. Incidence Rate and Multivariate Adjusted Hazard Ratios (HRs) during the 28-day Follow-up for Recurrence of Acute Cystitis in 2006-2007

\begin{tabular}{|c|c|c|c|c|c|c|c|c|}
\hline Recurrence of cystitis & $\mathrm{n}$ & Cases & Person-days & Incidence rate ${ }^{\mathrm{a}}$ & LR test ${ }^{b}$ & Crude HR (95\%CI) & $\begin{array}{l}\text { Adjusted } \\
\text { LR test }{ }^{c}\end{array}$ & $\begin{array}{c}\text { Adjusted HR } \\
(95 \% \mathrm{CI})\end{array}$ \\
\hline Adherence to & & & & & $<0.001$ & & $<0.001$ & \\
\hline \multicolumn{9}{|l|}{ Guidelines } \\
\hline Yes & 24,636 & 3,743 & 626,079 & 59.78 & & $0.89(0.85-0.94)^{* * *}$ & & $0.89(0.85-0.95)^{* * *}$ \\
\hline Sex & & & & & $<0.001$ & & $<0.001$ & \\
\hline Age (years) & & & & & $<0.001$ & & $<0.001$ & \\
\hline $18-29$ & 7,496 & 1,072 & 191,638 & 55.94 & & 1.00 & & 1.00 \\
\hline $30-39$ & 13,505 & 2,120 & 342,036 & 61.98 & & $1.11(1.03-1.19)^{* * *}$ & & $1.09(1.01-1.17)^{*}$ \\
\hline $50-64$ & 7,991 & 1,306 & 201,175 & 64.92 & & $1.16(1.07-1.25)^{* * *}$ & & $1.14(1.05-1.25)^{* * *}$ \\
\hline$\geq 65$ & 7,403 & 1,233 & 186,842 & 65.99 & & $1.17(1.08-1.27)^{* * *}$ & & $1.14(1.02-1.27)^{* *}$ \\
\hline No & 36,360 & 5,724 & 920,821 & 62.16 & & 1.00 & & 1.00 \\
\hline Yes & 35 & 7 & 870 & 80.46 & & $1.27(0.60-2.66)$ & & $1.17(0.56-2.46)$ \\
\hline Renal Diseases & & & & & 0.81 & & 0.80 & \\
\hline No & 35,893 & 5,650 & 908,964 & 62.16 & & 1.00 & & 1.00 \\
\hline Yes & 502 & 81 & 12,727 & 63.64 & & $1.03(0.82-1.28)$ & & $0.97(0.78-1.21)$ \\
\hline Urinary incontinence & & & & & 0.03 & & 0.08 & \\
\hline No & 34,711 & 5,433 & 879,490 & 61.77 & & 1.00 & & 1.00 \\
\hline Yes & 1,684 & 298 & 42,201 & 70.61 & & $1.14(1.01-1.28)^{*}$ & & $1.11(0.99-1.25)$ \\
\hline $\begin{array}{l}\text { Other Cardiovascular } \\
\text { diseases }\end{array}$ & & & & & 0.17 & & 0.31 & \\
\hline No & 29,408 & 4,593 & 745,169 & 61.64 & & 1.00 & & 1.00 \\
\hline Yes & 637 & 91 & 16,329 & 55.73 & & $0.90(0.49-1.67)$ & & $0.92(0.74-1.13)$ \\
\hline
\end{tabular}

a:Incidence rate: per 10,000 person-days

LR test ${ }^{b}$ : the likelihood ratio test for models with and without the specified covariate

Adjusted LR test ${ }^{c}$ : adjusted for all variables

${ }^{*} \mathrm{p}<0.05,{ }^{*} \mathrm{p}<0.01, * *{ }^{*} \mathrm{p}<0.001$

Adjusted HR: adjusted for all variables

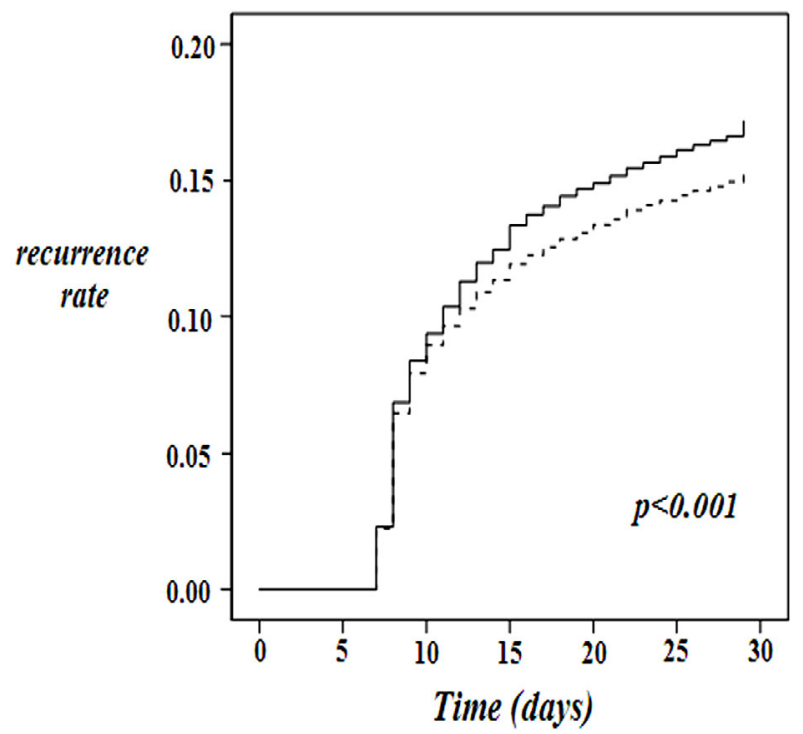

Figure 2. Comparison of cystitis recurrence rates of cystitis for adherence and nonadherence to antimicrobial prescription guidelines by log-rank test. The solid and dashed lines indicate the nonadherence and adherence groups, respectively. scriptions, $67.69 \%$ were guideline-recommended drugs that were prescribed during the patient's first visit for cystitis treatment. The frequencies of antibiotic prescriptions for patients with cystitis and their recurrence rate in Taiwan between 2006 and 2007 are listed in Table 3. The percentages of first-generation cephalosporin, fluoroquinolone, and trimethoprim prescribed were $31.61 \%, 15.32 \%$, and $7.04 \%$, respectively, and those were the most frequently prescribed drugs in the guideline-recommended group. On the other hand, using more than two antimicrobials for acute cystitis $(13.56 \%)$ was the most common inappropriate drug therapy.

\section{Discussion}

To the best of our knowledge, this is the first nationwide population-based follow-up study to examine the risk of recurrence among acute cystitis patients given inappropriate antimicrobial treatment. Our results indicated that patients with acute cystitis who received antimicrobials that were nonadherent to national guidelines (over- or underuse) had a significantly higher risk of recurrence than patients using ad- 
Table 3. Frequency Analysis of Antibiotics Prescribed for Patients with Cystitis and Their Recurrence Rate in Taiwan in 2006-2007 $(n=36,395)$

\begin{tabular}{lccrrr}
\hline Drugs & $\mathrm{n}$ & $\%$ & $\begin{array}{c}\text { Recurrent } \\
\text { cases }\end{array}$ & Person-days & Incidence $(95 \% \mathrm{CI})^{\mathrm{a}}$ \\
\hline Guideline-recommended drugs & 24,636 & 67.69 & 3,743 & 626,079 & $59.78(44.63-74.93)$ \\
1st G cephalosporin & 11,504 & 31.61 & 1,716 & 292,869 & $58.59(43.59-73.59)$ \\
Quinolone & 5,575 & 15.32 & 902 & 140,620 & $64.14(48.44-79.84)$ \\
$\quad$ Trimethoprim & 2,562 & 7.04 & 373 & 65,401 & $57.03(42.23-71.83)$ \\
Other drugs & 2,538 & 6.97 & 408 & 64,152 & $63.60(47.97-79.23)$ \\
Aminopenicillin & 2,439 & 6.70 & 340 & 62,609 & $54.31(39.87-68.75)$ \\
Trimethoprim/Sulfamethol & 18 & 0.05 & 4 & 428 & $93.46(74.51-112.41)$ \\
& & & & & \\
Non-guideline-recommended & 11,759 & 32.31 & 1,988 & 295,612 & $67.25(51.18-83.32)$ \\
drugs & & & & & \\
More than two & 4,936 & 13.56 & 857 & 123,945 & $69.14(52.84-85.44)$ \\
antimicrobials & 3,868 & 10.63 & 616 & 97,741 & $63.02(47.46-78.58)$ \\
Sulfamethol & 1,450 & 3.98 & 284 & 35,611 & $79.75(62.25-97.25)$ \\
Other drug & 894 & 2.46 & 139 & 22,770 & $61.05(45.74-76.36)$ \\
Lincomycin & 541 & 1.49 & 74 & 13,864 & $53.38(39.06-67.70)$ \\
Chloramphenicol & 70 & 0.19 & 18 & 1,681 & $107.08(86.80-127.36)$ \\
$\quad$ Aminoglycoside & & & & &
\end{tabular}

herent antimicrobials, even after adjusting for multiple chronic underlying diseases. Overall, first-generation cephalosporins were the most commonly prescribed antibiotics among guideline-recommended drugs, and sulfamethoxazole was the most frequently prescribed single antimicrobial among the non-guideline-recommended drugs.

UTIs, especially acute cystitis, are one of the most frequent diseases requiring antimicrobial treatment in ambulatory patients. Acute cystitis is mainly observed in women, and most of the patients in this study were women $(83 \%)$. The recurrence rate of cystitis is relatively high. Lawrenson et al. demonstrated that $14 \%$ of 75,045 newly treated patients required a second antibiotic therapy within 28 days of cystitis treatment for cystitis (15). These data are compatible with the results of our study; we found an overall $15.7 \%$ recurrence rate in 36,395 newly diagnosed patients, and patients prescribed non-guideline-recommended antimicrobials had a significantly higher risk of recurrence $(16.91 \%)$.

The high rate of acute cystitis recurrence after antimicrobial therapy was mostly due to the high resistance rates of uropathogens (16). The therapeutic management of lower UTIs has been hampered worldwide by the increasing resistance of uropathogens to commonly used antibiotics. The most common pathogens of cystitis are similar around the world; $80-90 \%$ of cases are caused by Escherichia coli (E. coli), and less frequent pathogens are Klebsiella pneumoniae and Proteus mirabilis (17). The Latin American SENTRY Program reported that the extended spectrum beta-lactamase (ESBL)-producing rates of E. coli and Klebsiella spp. have increased to $48.4 \%$ and $60.4 \%$, respectively (9). Nevertheless, carbapenemase-producing $K$. pneumoniae significantly increased in 2010 with clonal expansion in Latin American hospitals (18). Increased resistance has also been noted in Taiwan; the Taiwan Surveillance of Antimicrobial Resistance (TSAR) found that $124(52.8 \%)$ cases of ESBL-producing
K. pneumoniae were resistant to ciprofloxacin in the TSARIII program (10). Considering the patterns of high resistance, appropriately chosen antibiotics will reduce the recurrence rate of UTIs, thereby lowering the associated complications and costs. Thus, many countries and regions have established their own antimicrobial guidelines for acute cystitis therapy, including the European Association of Urology (EAU) guidelines and the Nederlands Huisartsen Genootschap (NHG)-Standard Urine Infection in the Netherlands $(2,19)$. An antibiotic treatment profile of acute cystitis in Taiwan (14) was established in 2000 and posted on the website of Department of Health as a reference to all practicing physicians in Taiwan. The guidelines demonstrated antimicrobial choices for uncomplicated and complicated UTIs depending upon the various characteristics of these patients. The influencing factors included age, infection site, and pregnancy status. Several studies have evaluated the contributing factors associated with local physicians' adherence to UTI treatment guidelines in many health care settings. The risk factors include age and gender of both the patients and physicians, and level and location of the hospitals (20). To date, no study has addressed the relationship between acute cystitis recurrence and antimicrobial treatments that do not adhere to national guidelines.

Although some previous studies have investigated women receiving norfloxacin treatment, diabetic patients have a significantly higher recurrence rate than nondiabetic controls (16.1 vs. $12.2 \%, \mathrm{p}=0.003$ ) (21). However, our study revealed that diabetes and other comorbidities were not significant risk factors for acute cystitis recurrence after multivariate adjustments. These findings support our hypothesis that adherence to the recommended guidelines can reduce the acute cystitis recurrence rate, regardless of whether patients have comorbidities.

According to the EAU classification system, sex and old 
age are possible risk factors taken into consideration (22). Similarly, a female sex and old age were significant factors for a poor clinical outcome in our study. Sex is already addressed in Taiwan's guidelines; however, age should also be added to these guidelines.

In general, the combination of sulfamethoxazole and trimethoprim (folic acid-synthesis blockers) is considered appropriate for the treatment or prophylaxis of UTI $(15,23)$. The clinical efficacy of trimethoprim alone for three days $(90 \%)$ is similar to the cotrimoxazole (trimethoprimsulfamethoxazole) regimen for three days (93\%) (24), but the prescription of sulmethoxazole as the sole therapeutic antibiotic may be inappropriate. No previous study has used sulmethoxazole as the only antibiotic treatment for a UTI. In our findings, sulmethoxazole was the most frequently drug used in the nonadherent treatment group, but the failure rate of patients treated with sulmethoxazole alone was $15.93 \%$, which was higher than the overall failure rate in the adherence to guideline treatment group (15.19\%). Thus, the spectrum of treatment efficiencies between cotrimoxazole and sulmethoxazole alone needs further clarification.

One study observed that $12-16 \%$ of young women with UTIs return for further treatment within 28 days, irrespective of the initial antibiotic prescribed (18). However, the recurrence rate was significantly higher in patients receiving guideline-adherent antimicrobials $(15.19 \%$ vs. $16.91 \%)$. This indicates that patients would have a good clinical outcome and low recurrence rate if physicians would adhere to guidelines and prescribe appropriate antimicrobials for acute cystitis. The results of our study can help policy-makers understand the variable prescribing behavior patterns among physicians, which could decrease the frequency of inappropriate antibiotic prescriptions and the cystitis recurrence rate. In addition, these findings may provide useful reasons to implement strategies that increase adherence to antimicrobial stewardship.

The longitudinal database and large population size for obtaining newly diagnosed cases with acute cystitis are the main strengths of this study. However, this study had several limitations. First, because the LHID 2005 is a secondary database of claims data for healthy patients with insurance, we could not obtain urine culture reports, including bacteria identification and antimicrobial resistance tests, to demonstrate the relationship between bacterial resistance patterns and antimicrobial treatment recurrence. Second, the risk behaviors for acute cystitis development in the two patient groups were also unavailable with regard to the frequencies of sexual activity, sexual intercourse, and cranberry consumption. Third, other risk factors concerning hospital factors, physician factors, and patient socioeconomic status could not be obtained from the databank.

In conclusion, physicians who adhered to the recommended guidelines for the treatment of bacterial infections achieved better therapeutic outcomes in their patients with acute cystitis, regardless of whether they had multiple chronic comorbidities. Thus, intensive implementation by physicians in all health care disciplines that treat patients with lower UTIs is necessary to ensure a discreet antibiotic policy that will decrease recurrence rates and improve patient care.

The authors state that they have no Conflict of Interest (COI).

\section{References}

1. Stamm WE, Hooton TM. Management of urinary tract infections in adults. N Engl J Med 329: 1328-1334, 1993.

2. Naber KG, Bergman B, Bishop MC, et al. Urinary Tract Infection (UTI) Working Group of the Health Care Office (HCO) of the European Association of Urology (EAU). Eur Urol 40: 576-588, 2001.

3. Armstrong EP. Clinical and economic outcomes of an ambulatory urinary tract infection disease management program. Am J Manag Care 7: 269-280, 2001.

4. Gorbach SL, Bartlett JG, Blacklow NR. Infectious Diseases: Urinary Tract Infection. 3rd ed. Lippincott Williams and Wilkins, Philadelphia, 2004: 861-869.

5. Hsu LY, Tan TY, Tam VH, Kwa A, Fisher DA, Koh TH. Surveillance and correlation of antibiotic prescription and resistance of Gram-negative bacteria in Singaporean hospitals. Antimicrob Agents Chemother 54: 1173-1178, 2006.

6. Wattal C, Joshi S, Sharma A, Oberoi JK, Prasad KJ. Prescription auditing and antimicrobial resistance at a tertiary care hospital in New Delhi, India. J Hosp Infect 59: 156-158, 2005.

7. Gales AC, Sader HS, Jones RN. Urinary tract infection trends in Latin American hospitals: report from the SENTRY antimicrobial surveillance program (1997-2000). Diagn Microbiol Infect Dis 44: 289-299, 2002.

8. Turnidge J, Bell J, Biedenbach DJ, Jones RN. Pathogen occurrence and antimicrobial resistance trends among urinary tract infection isolates in the Asia-Western Pacific Region: report from the SENTRY Antimicrobial Surveillance Program, 1998-1999. Int J Antimicrob Agents 20: 10-17, 2002.

9. Gales AC, Castanheira M, Jones RN, Sader HS. Antimicrobial resistance among Gram-negative bacilli isolated from Latin America: results from SENTRY Antimicrobial Surveillance Program (Latin America, 2008-2010). Diagn Microbiol Infect Dis 73: 354360, 2012.

10. Lin CJ, Siu LK, Ma L, Chang YT, Lu PL. Molecular epidemiology of ciprofloxacin-resistant extended-spectrum $\beta$-lactamaseproducing Klebsiella pneumoniae in Taiwan. Microb Drug Resist 18: 52-58, 2012.

11. Sheu JJ, Kang JH, Lin HC, Lin HC. Hyperthyroidism and risk of ischemic stroke in young adults: a 5-year follow-up study. Stroke 41: 961-966, 2010.

12. Cantrell R, Young AF, Martin BC. Antibiotic prescribing in ambulatory care settings for adults with colds, upper respiratory tract infections, and bronchitis. Clin Ther 24: 170-182, 2002.

13. Lawrenson RA, Logie JW. Antibiotic failure in the treatment of urinary tract infections in young women. J Antimicrob Chemother 48: 895-901, 2001.

14. Society I. Guidelines for antimicrobial therapy of urinary tract infections in Taiwan. J Microbiol Immunol Infect 33: 271-272, 2000.

15. Centers for Diseases Control, R.O.C. Guidelines for antimicrobial therapy of urinary tract infections in Taiwan. Available at: http:// www.idsroc.org.tw/db/health/3.pdf, 2000. (accessed 21 November, 2012).

16. Raz R, Chazan B, Kennes Y, et al. Empiric use of trimethoprimsulfamethoxazole (TMP-SMX) in the treatment of women with 
uncomplicated urinary tract infections, in a geographical area with a high prevalence of TMP-SMX-resistant uropathogens. Clin Infect Dis 34: 1165-1169, 2002.

17. Tice AD. Short-course therapy of acute cystitis: a brief review of therapeutic strategies. J Antimicrob Chemother 43 (Suppl A): 8593, 1999.

18. Castanheira M, Costello AJ, Deshpande LM, Jones RN. Expansion of clonal complex $258 \mathrm{KPC}-2$-producing Klebsiella pneumoniae in Latin American hospitals: report of the SENTRY Antimicrobial Surveillance Program. Antimicrob Agents Chemother 56: 1668$1669,2012$.

19. van Pinxteren B, van Vliet SM, Wiersma TJ, Goudswaard AN. Summary of the practice guideline 'Urinary-tract infections' (second revision) from the Dutch College of General Practitioners. Ned Tijdschr Geneeskd 150: 718-722, 2006.

20. Kahan NR, Friedman NL, Lomnicky Y, et al. Physician speciality and adherence to guidelines for the treatment of unsubstantiated uncomplicated urinary tract infection among women. Pharma- coepidemiol Drug Saf 14: 357-361, 2005.

21. Schneeberger C, Stolk RP, Devries JH, Schneeberger PM, Herings RM, Geerlings SE. Differences in the pattern of antibiotic prescription profile and recurrence rate for possible urinary tract infections in women with and without diabetes. Diabetes Care 31: 1380-1385, 2008.

22. Johansen TE, Botto $H$, Cek M, et al. Critical review of current definitions of urinary tract infections and proposal of an EAU/ ESIU classification system. Int J Antimicrob Agents 38 (Suppl): 64-70, 2011.

23. Gupta K, Hooton TM, Naber KG, et al. International clinical practice guidelines for the treatment of acute uncomplicated cystitis and pyelonephritis in women: A 2010 update by the Infectious Diseases Society of America and the European Society for Microbiology and Infectious Diseases. Clin Infect Dis 52: e103-e120, 2011.

24. Hooton TM. Clinical practice. Uncomplicated urinary tract infection. N Engl J Med 366: 1028-1037, 2012.

(C) 2014 The Japanese Society of Internal Medicine http://www.naika.or.jp/imonline/index.html 